\title{
Promoting continuity of care should be integral to any health care system
}

\author{
Walter Rosser MD, Karen Schultz MD
}

$\infty$

See related article page $\mathrm{I}_{3} 62$

M any physicians and patients believe that a long-term doctor-patient relationship built on trust and mutual respect is a central tenet of effective and efficient health care. ${ }^{1}$ However, are such pronouncements, frequently made by colleges representing primary-care disciplines, based on more than collective experience or ideals? Is there evidence that defines what elements of continuity of care are most enhanced by a long-term relationship? The complexity of these relationships raise many important questions for researchers and educators. What specific actions or components of the relationship benefit the patient most? Is it a detailed cumulative understanding of the patient's history? Is it an enhanced advocacy role by the physician? Is it a better understanding of the patient's values and expectations? Or is it smoother transition of care? Unfortunately, there are few solid answers to any of these and many other questions. Ask any family physician if continuity of care is an important feature of Canadian family practice and the answer will be Yes. The family physician will mention the importance of building trusting relationships with patients, especially those with chronic diseases or comorbidity. ${ }^{1}$ Most family physicians can provide examples of how their understanding and management of their patients' complex problems has resulted in reduced use of emergency departments and hospital beds. Many physicians are also likely to mention the high levels of satisfaction they have gained from their relationships with these patients.

The College of Family Physicians of Canada states that the doctor-patient relationship is central to the discipline of family medicine and is I of the 4 principles of family medicine that influences the education of both residents and practising family physicians. ${ }^{2}$ This statement is based on decades of experience of thousands of members providing care for Canadians and the benefits perceived to arise from a strong, trusting doctor-patient relationship.

Is there evidence to support the perceived benefits of continuity of care? Is it important for both physicians and their patients to be aware of the far-reaching impact of continuity of care, particularly as new models of health care delivery are being proposed? Evidence suggests that continuity of care improves the uptake of preventive care, enhances adherence to therapy, and increases patient and physician satisfaction. ${ }^{3}$ There is increasing evidence that patients' health status and chronic disease outcomes are improved with continuity of care. ${ }^{3}$ Most would consider these findings to be important outcomes. The cost-effectiveness of medical care has garnered much attention lately, which raises the question of whether continuity of care improves cost-effectiveness and decreases the number of visits to the emergency department and admissions to hospital.

In a study in the United States, Gill and colleagues ${ }^{4}$ showed that high provider continuity was associated with lower emergency department use among patients supported by Medicaid. Christakis and associates ${ }^{5}$ found similar results in a pediatric population in the United States, where increased continuity of care was associated with a decreased number of visits to the emergency department and admissions to hospital. The study by Ionescu-Ittu and colleagues ${ }^{6}$ in this issue of CMAJ makes an important contribution to improving our understanding of the impact of continuity of care. The authors examined data for a random sample of more than 95000 Quebec residents aged 65 or more. Unlike most previous studies, they used Quebec health insurance data to demonstrate that having a primary physician and continuity of care reduced the rate of emergency department use. The authors also highlight the importance of the relationship between doctor and patient rather than the physician's specialty. The term "primary physician" in their study, although made up of more than $80 \%$ family doctors, also included a number of specialists, since the authors explain that elderly patients with chronic diseases often see specialists regularly and consider them as their primary care physician.

Ionescu-Ittu and colleagues found that, during the 2-year study period, an increased rate of emergency department use was associated with a low or medium (v. high) level of continuity of care with the patient's primary physician (adjusted rate ratio I.46, 95\% confidence interval [CI] I.44-I.48, and I.27, 95\% CI I.25-I.29, respectively) and lack of a primary physician (adjusted rate ratio I.45, 95\% CI I.4I-I.49). They also found a dose-response relation between continuity of care and emergency department use among patients with a primary physician. The authors carefully selected indexes and definitions for each term they used in the study. Most studies of continuity of care have been identified as methodologically weak; however, 20 of 22 of these studies arrived at conclusions supporting continuity of care. ${ }^{7}$ To date, the study by IonescuIttu and colleagues ${ }^{6}$ makes the most significant contribution to a growing body of evidence demonstrating the benefits of continuity of care, especially in the elderly population.

During the past decade a number of changes in Canada's health care system have reduced the possibility for continuity of care. Examples include the increasing fragmentation of

Walter Rosser is Emeritus Professor of Family Medicine and Karen Schultz is Assistant Professor of Family Medicine at Queen's University, Kingston, Ont. 
health care delivery owing to the rising number of agencies and organizations delivering health care, use of specialty clinics for specific problems (e.g., varicose vein clinics, weight loss clinics, hair epalation clinics, Botox clinics), visits to anticoagulation clinics and visits to walk-in clinics not connected to family practices. Physician shortages in many parts of the country make continuity of care difficult or impossible for patients who do not have a regular physician.

Surely this growing body of evidence suggesting increased efficiency and effectiveness as a result of continuity of care should stimulate strategizing within Canadian provincial and territorial health insurance plans to promote continuity of care for elderly patients or patients who have chronic diseases. In Ontario, the Family Health Network and Family Health Team concepts use physician incentives, nurse practitioners, health educators, pharmacists and dietitians to promote improvement in care for both patients with chronic diseases and elderly patients with comorbid conditions. ${ }^{8}$ There are incentives promoting continuity of care with I physician or nurse practitioner. Whether continuity is as important for younger patients and for patients without chronic disease will require further studies. From a health systems viewpoint, the findings from the study by Ionescu-Ittu and colleagues have added importance because elderly patients are the heaviest users of the health care system and are rapidly growing in numbers. Reducing costs and improving efficiency in health care delivery for this age group has the potential of more cost-effective care.
The time has arrived for legitimizing the perceptions of generations of family physicians and building a health care system that values and promotes continuity of care.

Competing interests: None declared.

Contributors: Both of the authors substantially contributed to the conception and content of the article, drafted and revised the manuscript critically for important intellectual content and gave final approval of the version to be published.

\section{REFERENCES}

I. Rosser WW, Kasperski J. The benefits of a trusting physician-patient relationship. J Fam Pract 2001;50:329-30.

2. College of Family Physicians of Canada. Four principles of family medicine. Mississauga (ON): The College. Available: www.cfpc.ca/English/cfpc/about\%2ous /principles/default.asp?s=I (accessed 2007 Oct 5).

3. Gray DP, Evans P, Sweeney K, et al. Towards a theory of continuity of care. JR Soc Med 2003;96:160-6.

4. Gill JM, Mainous AG III, Nsereko M. The effect of continuity of care on emergency department use. Arch Fam Med 2000;9:333-8.

5. Christakis DA, Mell L, Koepsell TD, et al. Association of lower continuity of care with greater risk of emergency department use and hospitalization in children. Pediatrics 2001;107:524-9.

6. Ionescu-Ittu R, McCusker J, Ciampi A, et al. Continuity of primary care and emergency department utilization among elderly people. $C M A J$ 2007;177:1362-8.

7. Saultz JW, Albedaiwi W. Interpersonal continuity of care and patient satisfaction: a critical review. Ann Fam Med 2004;2:445-5I.

8. Lee RL. Family healthy networks in Ontario [letter]. CMAJ 2003;I68:152.

Correspondence to: Dr. Walter Rosser, Department of Family Medicine, Queen's University, 220 Bagot St., Kingston ON K>L 5E9; rosserw@post.queensu.ca 